\section{Vegetables associated with reduced risk of cancer}

\author{
Robert Skopec* \\ Researcher Analyst, Dubnik, Slovakia
}

\section{Abstract}

The present study aimed to investigate and identify the association between the intake of allium vegetables and colorectal cancer (CRC) in population. A hospital-based matched casecontrol study was conducted between June 2009 and November 2011 in three hospitals. Eight hundred thirty three consecutively recruited cases of CRC were frequency matched to 833 controls by age (within 2.5 years of difference), sex, and residence area (rural/urban). Demographic and dietary information were collected via face-to-face interviews using a validated food frequency questionnaire. Adjusted odds ratios (aORs) and 95\% confidence intervals (Cls) were estimated by using unconditional logistic regression.

\section{More Information}

*Address for Correspondence: Robert Skopec, Researcher-analyst, Dubnik, Slovakia, Email: zxcbnvm7@gmail.com

Submitted: 11 March 2020

Approved: 18 March 2020

Published: 19 March 2020

How to cite this article: Skopec R. Vegetables associated with reduced risk of cancer. Arch Cancer Sci Ther. 2020; 4: 007-014.

DOI: 10.29328/journal.acst.1001014

Copyright: @ 2020 Skopec R. This is an open access article distributed under the Creative Commons Attribution License, which permits unrestricted use, distribution, and reproduction in any medium, provided the original work is properly cited.

Keywords: Allium vegetables; Colorectal cancer; Demographic information; Dietary information; Decreased CRC risk; Total allium intake; Inversely risk; Distal colon cancer

\section{Check for updates}

(1) OPEn ACCESS
A decreased CRC risk was observed for the consumption of total (aORs of high total allium intake compared with low total allium intake $=0.21,95 \% \mathrm{CI}=0.14-0.30, p$ trend $<0.001$ ) and several individual allium vegetables including garlic, garlic stalks, leek, onion, and spring onion ( $p$ trend < 0.05). By further sex-stratified analysis, allium vegetable intake was demonstrated to be inversely associated with the risk of CRC in both men and women. However, the association of garlic intake with cancer risk was not significant among those with distal colon cancer $(\mathrm{aOR}=0.53,95 \% \mathrm{CI}=0.27-$ 1.05, $p$ trend $=0.248)[1,2]$.

\section{Materials and methods}

In this analysis in a Northeast Chinese population, both men and women that the consumption of allium vegetables is associated with a reduced risk of CRC, regardless of colonic tumor subsite, with the exception of garlic intake in distal colon cancer.

\section{How the humble cabbage can stop cancers}

Scientists say they have discovered why some vegetables - including cabbage, broccoli and kale-can reduce the risk of bowel cancers. That cruciferous veg is good for the gut has never been in doubt but a detailed explanation has been elusive $[3,4]$.

The team at the Francis Crick Institute found anti-cancer chemicals were produced as the vegetables were digested. Cancer Research UK said there were plenty of reasons to eat more veg. The work focused on how vegetables alter the lining of the intestines, by studying mice and miniature bowels growing in the lab.

Like the skin, the surface of the bowels is constantly being regenerated in a process that takes four to five days. But this constant renewal needs to be tightly controlled, otherwise it could lead to cancer or gut inflammation. And the work, published in the journal Immunity. Showed chemicals in cruciferous vegetables were vital.

\section{From kitchen to cancer prevention?}

The researchers investigated a chemical called indole3-carbinol, which is produced by chewing such vegetables. "Make sure they're not overcooked, no soggy broccoli," said researcher Dr Gitta Stockinger [5,6].

The chemical is modified by stomach acid as it continues its journey through the digestive system. In the lower bowel, it can change the behavior of stem cells, which regenerate the bowel lining, and of immune cells that control inflammation.

The study showed diets high in indole-3-carbinol protected the mice from cancer, even those whose genes put them at very high risk of the disease. Without the protective diet, the gut cells divided uncontrollably.

Dr. Stockinger added: "Even when the mice started 
developing tumours and we switched them to the appropriate diet, it halted tumour progression" $[7,8]$.

Signs of bowel cancer include persistent:

- blood in the stools

- changes in bowel habits, such as going to the toilet more often

- tummy pain, bloating or discomfort

Dr. Stockinger said the findings were a "cause for optimism". She has reduced the amount of meat she eats and now consumes a lot more vegetables.

She told the BBC: "A lot of dietary advice we're getting changes periodically - it is very confusing and not clear cut what the causes and consequences are." Just telling me it's good for me without a reason will not make me eat it.

"With this study, we have the molecular mechanisms about how this system works."

Prof. Tim Key, from Cancer Research UK, said: "This study in mice suggests that it's not just the fibre contained in vegetables like broccoli and cabbage that help reduce the risk of bowel cancer, but also molecules found in these vegetables too.

"Further studies will help find out whether the molecules in these vegetables have the same effect in people, but in the meantime there are already plenty of good reasons to eat more vegetables."

\section{Genetic variants which predispose women to endometrial cancer}

The systematic review, led by Professor Emma Crosbie from The University of Manchester, could help scientists develop targeted screening and prevention strategies for women at greatest risk of the disease $[9,10]$.

Although each genetic variant changes cancer risk by a small fraction, when all 24 variants are combined in a socalled polygenic risk score, women who score within the top $1 \%$ have a risk of endometrial cancer 3.16 times higher than the mean risk they say.

Published in the Journal of Medical Genetics, the study is the most comprehensive systematic review carried out to critically appraise the evidence on genetic variants implicated in predisposition to endometrial cancer.

The team was based at The University of Manchester, University of Cambridge and Manchester University NHS Foundation Trust and was funded by the NIHR Manchester Biomedical Research Centre [11,12].

The research is an important milestone in the study of endometrial cancer, the sixth most commonly occurring cancer in women and the $15^{\text {th }}$ most commonly occurring cancer overall according to the World Cancer Research Fund.

In the UK there are about 8,600 new cases per year according to Cancer Research UK. The team searched 2,674 abstracts, narrowing them down to 149 papers which were eligible for inclusion in the study. Specifically, genetic variants in HNF1B, KLF, EIF2AK, CYP19A1, SOX4 and MYC were strongly associated with the cancer.

Nineteen variants were reported with genome-wide significance and a further five with suggestive significance. They found no convincing evidence for the widely studied MDM2 variant rs2279744 as a risk factor.

"Because many of the studies carried out to date have been of variable quality, we felt it was important to understand more fully genetic predispositions to endometrial cancer," said Professor Crosbie. "Our work, we hope, will facilitate personalized risk assessment so that prevention and screening could be targeted more efficiently."

She added: "These genetic variants linked to endometrial cancer risk are involved in cell survival, estrogen metabolism and transcriptional control -- when the information stored in our DNA is converted into instructions for making proteins or other molecules $[13,14]$.

"We think studies with larger cohorts are needed to identify more variants with genome-wide significance."But until data from larger and more diverse cohorts are available, these twenty-four SNPs are the most robust common genetic variants that affect endometrial cancer risk."

\section{Converting cancer cells to fat cells to stop cancer's spread}

A method for fooling breast cancer cells into fat cells has been discovered by researchers from the University of Basel. The team were able to transform EMT-derived breast cancer cells into fat cells in a mouse model of the disease - preventing the formation of metastases. The proof-of-concept study was published in the journal Cancer Cell.

Malignant cells can rapidly respond and adapt to changing micro environmental conditions, by reactivating a cellular process called epithelial-mesenchymal transition (EMT), enabling them to alter their molecular properties and trans differentiate into a different type of cell (cellular plasticity).

Senior author of the study Gerhard Christofori, professor of biochemistry at the University of Basel, commented in a recent press release:

"The breast cancer cells that underwent an EMT not only differentiated into fat cells, but also completely stopped proliferating."

"As far as we can tell from long-term culture experiments, the cancer cells-turned-fat cells remain fat cells and do not revert back to breast cancer cells," he explained. 


\section{Epithelial-mesenchymal transition and cancer}

Cancer cells can exploit EMT-a process that is usually associated with the development of organs during embryogenesis-in order to migrate away from the primary tumor and form secondary metastases. Cellular plasticity is linked to cancer survival, invasion, tumor heterogeneity and resistance to both chemo and targeted therapies. In addition, EMT and the inverse process termed mesenchymal-epithelial transition (MET) both play a role in a cancer cell's ability to metastasize $[15,16]$.

Using mouse models of both murine and human breast cancer the team investigated whether they could therapeutically target cancer cells during the process of EMT-whilst the cells are in a highly plastic state. When the mice were administered Rosiglitazone in combination with MEK inhibitors it provoked the transformation of the cancer cells into post-mitotic and functional adipocytes (fat cells). In addition, primary tumor growth was suppressed and metastasis was prevented.

\section{Christofori highlights the two major findings in the study}

"Firstly, we demonstrate that breast cancer cells that undergo an EMT and thus become malignant, metastatic and therapy-resistant, exhibit a high degree of stemness, also referred to as plasticity. It is thus possible to convert these malignant cells into other cell types, as shown here by a conversion to adipocytes."

"Secondly, the conversion of malignant breast cancer cells into adipocytes not only changes their differentiation status but also represses their invasive properties and thus metastasis formation and their proliferation. Note that adipocytes do not proliferate anymore, they are called 'post-mitotic', hence the therapeutic effect."

Since both drugs used in the preclinical study were FDAapproved the team are hopeful that it may be possible to translate this therapeutic approach to the clinic. "Since in patients this approach could only be tested in combination with conventional chemotherapy, the next steps will be to assess in mouse models of breast cancer whether and how this transdifferentiation therapy approach synergizes with conventional chemotherapy. In addition, we will test whether the approach is also applicable to other cancer types. These studies will be continued in our laboratories in the near future."

\section{Processed foods may hold key to rise in autism}

In the lab, UCF scientists found exposing neural stem cells to excessive PPA damages brain cells in several ways $[17,18]$.

With the number of children diagnosed with autism on the rise, the need to find what causes the disorder becomes more urgent every day. UCF researchers are now a step closer to showing the link between the food pregnant women consume and the effects on a fetus' developing brain.
Saleh Naser, Latifa Abdelli and UCF undergraduate research assistant Aseela Samsam have identified the molecular changes that happen when neuro stem cells are exposed to high levels of an acid commonly found in processed foods. In a study published in Scientific Reports, a Nature journal, the UCF scientists discovered how high levels of Propionic Acid (PPA), used to increase the shelf life of packaged foods and inhibit mold in commercially processed cheese and bread, reduce the development of neurons in fetal brains.

Naser, who specializes in gastroenterology research at the College of Medicine's Burnett School of Biomedical Sciences, began the study after reports showed that autistic children often suffer from gastric issues such as irritable bowel syndrome. He wondered about a possible link between the gut and the brain and began examining how the microbiome - or gut bacteria - differed between people with autism and those who do not have the condition $[19,20]$.

"Studies have shown a higher level of PPA in stool samples from children with autism and the gut microbiome in autistic children is different," Naser says. "I wanted to know what the underlying cause was."

In the lab, the scientists found exposing neural stem cells to excessive PPA damages brain cells in several ways. First, the acid disrupts the natural balance between brain cells by reducing the number of neurons and over-producing glial cells. While glial cells help develop and protect neuron function, too many glia cells disturb connectivity between neurons. They also cause inflammation, which has been noted in the brains of autistic children.

Excessive amounts of the PPA acid also shorten and damage pathways that neurons use to communicate with the rest of the body. The combination of reduced neurons and damaged pathways impede the brain's ability to communicate, resulting in behaviors that are often found in children with autism, including repetitive behavior, mobility issues and inability to interact with others.

Previous studies have proposed links between autism and environmental and genetic factors, but Drs. Naser and Abdelli say their study is the first to discover the molecular link between elevated levels of PPA, proliferation of glial cells, disturbed neural circuitry and autism. The 18-month study was self-funded by UCF.

PPA occurs naturally in the gut and a mother's microbiome changes during pregnancy and can cause increases in the acid. But Drs. Naser and Abdelli says eating packaged foods containing the acid can further increase PPA in the woman's gut, which then crosses to the fetus.

More research needs to be done before drawing clinical conclusions. Next, the research team will attempt to validate its findings in mice models by seeing if a high PPA maternal 
diet causes autism in mice genetically predisposed to the condition. There is no cure for autism, which affects about 1 in 59 children, but the scientists hope their findings will advance studies for ways to prevent the disorder $[20,21]$.

"This research is only the first step towards better understanding of Autism Spectrum Disorder," the UCF scientists concluded. "But we have confidence we are on the right track to finally uncovering autism etiology."

\section{Scientists stop breast cancer cells from spreading}

Biologists have discovered a way to stop cells from one of the most aggressive types of breast cancer spreading in the lab. The study points towards new avenues of research to combat the devastating disease.

The results of the study of triple-negative breast cancer by the team from the Universities of Manchester, Glasgow and Sheffield and funded by Breast Cancer Now are published in Oncogene.

Because the cancer does not respond to hormonal therapies or targeted treatments used in other forms of the disease, the search is on for scientists to find other medications.

Around $15 \%$ of all breast cancers are triple negative, which are more likely to spread beyond the breast and more likely to recur after treatment $[22,23]$.

It is more common in younger people, African-American and Hispanic women and people who carry a BRCA1 mutation.

Using cutting edge genetic editing CRISPR technology, the team were able to delete either of two proteins-RUNX2 and CBF beta.

Removing either stopped the cancer from spreadingthough the absence of CBF beta seemed to have the more powerful effect. By putting either of the deleted proteins back into the cells, the cancer started to spread again.

Dr. Paul Shore from The University of Manchester was the lead researcher on the project. He said: "Triple-negative breast cancer is a particularly devastating disease which doctors, scientists and patients are investing much time and effort into finding new and better treatments.

"So this discovery is an important milestone in the understanding of how metastatic cancer spreads-though clearly, there's a long way to go before it has a chance of being translated into effective therapies.

"But nevertheless, by finding a way to intervene in this two-protein mechanism, it could be a possible drug target-and that could even have a relevance to other cancers as well."

He added: "We must bear in mind that these two proteins are found in other healthy tissues as well, so compounds will need to find a way to avoid them.
"And it is important to point out that we don't yet know if this effect is true for all Triple Negative cells - as there are many phenotypes of this particular disease."

Dr. Kotryna Temcinaite, Research Communications Manager at Breast Cancer Now, which funded the study, said: "These promising findings help us to understand more about how some triple negative breast cancers spread around the body and become incurable. We hope that the discovery of the role of the $\mathrm{CBF}$ beta protein in the spread of the disease could one day lead to much-needed new treatment options.

"Around 8,000 women in the UK each year are diagnosed with triple negative breast cancer, which can be more aggressive and more likely to come back and spread in the first few years after treatment. With these women still severely lacking in targeted therapies, it remains one of the greatest areas of unmet need in breast cancer $[23,24]$.

"We now look forward to further studies to help us fully understand the role that this and other proteins play in helping breast cancer spread, and to uncover whether we could target them with drugs to treat the disease more effectively. In the meantime, anyone who is concerned about the risk of their breast cancer returning or spreading can call our free Helpline on 08088006000 ."

\section{Protein could offer target for cancer metastasis}

A new study by University of Kentucky Markey Cancer Center researchers suggests that targeting a protein known as heat shock protein 47 (Hsp47) could be key for suppressing breast cancer metastasis $[15,17]$.

Metastasis is when cancer cells spread from the primary tumor to surrounding tissues and distant organs in the body and is the primary cause for breast cancer mortality. It is estimated that metastasis is responsible for about $90 \%$ of breast cancer deaths.

The study, led by Markey researcher Ren Xu, associate professor in the UK College of Medicine's Department of Pharmacology and Nutritional Sciences, found that Hsp47, a protein that assists with collagen production, plays a role in breast cancer metastasis.

$\mathrm{Xu}$ and co-author Gaofeng Xiong analyzed DNA/RNA generated from human breast cancer tissue, which showed that increased production of Hsp47 was linked to metastasis in triple-negative breast cancer. About 10\% - 20\% of breast cancers are classified as triple-negative, which is considered to be more aggressive and have a poorer prognosis than other types of breast cancer.

The results suggest that targeting Hsp47 could be a promising strategy to block cancer cell-platelet interaction and cancer colonization in secondary organs for triplenegative breast cancer patients, says Xu. 
"If we can find a way to target this pathway with therapeutics we could block this process and therefore inhibit metastasis," Xu said.

Xu says another benefit of the study is the potential that Hsp47 could serve as a prognosis or biomarker of metastasis since it is often amplified in the genes of metastatic breast cancer tissue.

\section{Red meat, processed meat and cancer}

Did you know that eating more than 700 grams (raw weight) of red meat a week increases your risk of bowel cancer? Or that the risk of developing bowel cancer goes up 1.18 times for every 50 grams of processed meat eaten per day?

The World Health Organization has classified processed meats including ham, bacon, salami and frankfurts as a Group 1 carcinogen (known to cause cancer) which means that there's strong evidence that processed meats cause cancer. Eating processed meat increases your risk of bowel and stomach cancer. Red meat, such as beef, lamb and pork, has been classified as a Group 2A carcinogen which means it probably causes cancer.

\section{Red and processed meat increase cancer risk}

Current research shows that there are certain chemicals in red and processed meats - both added and naturally occurring - that cause these foods to be carcinogenic. For example, when a chemical in red meat called haem is broken down in the gut, $\mathrm{N}$-nitroso chemicals are formed and these have been found to damage the cells that line the bowel, which can lead to bowel cancer. These same chemicals also form when processed meat is digested. In addition, the nitrite and nitrate preservatives used to preserve processed meat produce these N-nitroso chemicals and can lead to bowel cancer. To find out your cancer risk, take the Cancer Risk Quiz.

\section{Cancer council recommendations}

To reduce your risk of cancer, Cancer Council recommends eating no more than 1 serve of lean red meat per day or 2 serves 3-4 times per week. Red meat includes beef, lamb and pork.

Cut out processed meats altogether or keep them to an absolute minimum. Processed meats include bacon, ham, devon, frankfurts, chorizo, cabanossi and kransky.

\section{Cutting down on red and processed meats will reduce your cancer risk}

Vegetarian diets can be healthy and balanced too. However, if you don't eat meat or other animal foods, it is important to ensure you are getting enough protein, Vitamin B12, iron, zinc, calcium and omega 3 fatty acids.
Examples of serve sizes:

o 1 serve = 1 lamb loin chop (about 100g raw)

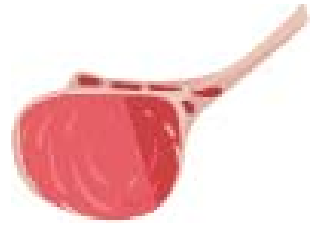

o 2 serves = 1 medium porterhouse $/$ scotch fillet steak (about 200g raw)

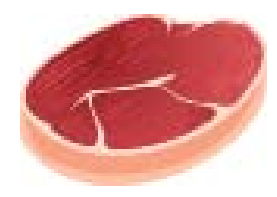

o 2 serves $=1$ cup diced meat (about 200g raw)

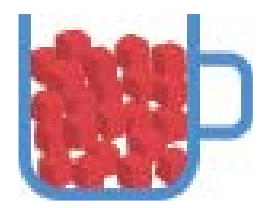

\section{Tips to reduce your cancer risk}

Cut out processed meat. Try swapping the prosciutto and pepperoni out of your pizza or pasta for chicken, mushrooms, eggplant, or capsicum.

Reduce your red meat portions. Try marinated chicken or swap meat for fish a couple of times each week.

Add eggs, beans, lentils, fish or chicken to your meals for a protein boost.

Try a vegie pattie burger, vegetable lasagne, tofu stir fry or a falafel wrap.

\section{Masterswitch discovered in body's immune system}

Scientists have discovered a critical part of the body's immune system with potentially major implications for the treatment of some of the most devastating diseases affecting humans.

Professor Graham Lord, from The University of Manchester, led the study, which could translate into treatments for autoimmune diseases including Cancer, Diabetes, Multiple.

\section{Sclerosis and crohn's disease within a few years}

The discovery of the molecular pathway regulated by a tiny molecule-known as microRNA-142 is a major advance in our understanding of the immune system.

The 10-year-study found that microRNA-142 controls Regulatory $\mathrm{T}$ cells, which modulate the immune system and prevent autoimmune disease. It is, they found, the most highly expressed regulator in the immune system. Professor Lord, led 
the research while at Kings College London in collaboration with Professor Richard Jenner at UCL.

And according to Professor Lord, the discovery could be translated into a viable drug treatment within a few years.

He said: "Autoimmune diseases often target people in the prime of their life creating a significant socio-economic burden on them. Sometimes, the effect can be devastating, causing terrible hardship and suffering.

"But these findings represent a significant step forward in the understanding of the immune system and we believe many people worldwide may benefit."

If the activity of Regulatory $\mathrm{T}$ cells is too low, this can cause other immune cells to attack our own body tissues. If these Regulatory $\mathrm{T}$ cells are too active, this leads to suppression of immune responses and can allow cancers to evade the immune system.

So being able to control them is a major step forward in our ability to control- and harness-the therapeutic power of the immune system.

Professor Richard Jenner from UCL, who led the computational side of the project, said that: "We were able to trace the molecular fingerprints of this molecule across other genes to determine how it acted as such a critical regulator."

Professor Lord, now Vice President and Dean of the Faculty of Biology, Medicine and Health at The University of Manchester, added: "Scientists over the past decade or so have developed therapies which are able to modulate different pathways of the immune system. We hope that this new discovery will lead to the development of new ways to treat autoimmunity, infectious diseases and cancer and we are incredibly excited about where this may lead" $[17,18]$.

\section{A new way to get 'forever chemicals' linked to cancer out of our water}

Researchers at Clarkson University are working with the US Air Force to remove a group of "forever chemicals" from water. The chemicals, known as PFAS, have been associated with cancer, liver damage, and developmental issues. They're found in food packaging, cookware, outdoor gear, and firefighting foam. The researchers found a way to zap the chemicals in a plasma reactor, effectively destroying the bond that allows them to stay in the body for life.

More than 70 years ago, a group of chemicals known as PFAS promised to make people's lives easier and more efficient. The category of chemicals-whose full name is perand polyfluoroalkyl substances-was developed in the $1940 \mathrm{~s}$ to resist heat, grease, stains, and water. That made them ideal coatings for food packaging, paper plates, and cookware.
They were also used as firefighting foam for military training exercises and emergency responses starting in the 1970s. But since then, scientists have uncovered links between PFAS and cancer, liver damage, thyroid disease, and developmental issues.

Today, the chemicals are in the bloodstreams of $99 \%$ of Americans, The Intercept reported. They've been found at more than 700 sites across 49 US states, according to EWG.

PFAS can linger in water and air for thousands of years, so consuming or inhaling them means they could stay in the body for life-hence their nickname, "forever chemicals."

But a group of researchers at Clarkson University in New York is developing a way to destroy PFAS in water. Together with the US Air Force, the team is using machines called plasma reactors to sever the chemicals' carbon-fluorine bonds-the same bonds that make them virtually indestructible in the environment.

The technology applies only to PFAS in groundwater. (The researchers are working on a separate project to remove PFAS from soil.) But it could eventually be cheaper than the current water-filtration process $[19,21]$.

\section{Scientists are splitting PFAS molecules apart}

The Air Force began using a new firefighting foam that doesn't contain PFAS in July 2018, but it still has to contend with the legacy of the old one. The chemicals have gotten into the groundwater at numerous Air Force bases across the US, forcing the military to spend more than $\$ 2.2$ billion to clean up PFAS-contaminated sites, according to a report on the Environmental Protection Agency's website.

That's why the Air Force is looking for a cheaper way to clean contaminated water.

To test their technology, the Clarkson researchers built a 20 -foot-long mobile trailer that holds two plasma reactors. For two weeks in September, they pumped PFAS-contaminated groundwater from the Wright-Patterson Air Force Base in Ohio into the reactors.

Argon gas at the bottom of the reactors carries the PFAS molecules to the surface. The researchers then use highvoltage electrodes to generate plasma, an ionized gas made of free-roaming electrons and positive ions.

The plasma zaps the water's surface, where it spreads across like fire, hitting the PFAS molecules and splitting them apart. Once the carbon and fluorine molecules have been separated, the PFAS compound is effectively destroyed.

Selma Mededovic, the principal researcher overseeing the project, said treating a single gallon of water this way takes one minute. 
That's far slower than one of the standard methods for removing PFAS: adding carbon to contaminated water. With that approach, the chemicals stick to carbon, allowing clean water to be filtered out. Hundreds of gallons of water per minute can be treated this way, but Mededovic said the spent carbon needed to be incinerated afterward.

That incineration process costs about $\$ 3$ to $\$ 4$ per gallon of contaminated water, she said. Her plasma method, by contrast, is at least $40 \%$ cheaper (though the estimate does not include the cost of the reactors).

Mededovic said she would have a better sense of how much the reactors cost once the first commercial prototype is built. She expects it to be operational by the end of this year.

In the meantime, her team is ramping up the technology to treat 15 gallons of contaminated water per minute. Eventually, they hope to treat about 200 gallons per minute.

"We are working on scaling up our process to be competitive to carbon," Mededovic said.

\section{The EPA doesn't have a legal limit for PFAS in water}

The Clarkson team's plasma reactor reduces PFAS concentrations in water well below the EPA's general limit for drinking-water contaminants: 70 parts per trillion.

But environmental groups have expressed concern that the limit is too high when it comes to PFAS. (The nonprofit Environmental Working Group endorses a limit of one part per trillion) $[9,10]$.

The EPA pledged to develop national drinking-water regulations for PFAS by the end of 2019, but the deadline came and went.

On Friday, the US House of Representatives passed a group of measures that would require the EPA to set that guideline. The measures would also label PFAS as "hazardous substances," which would allow the EPA to require industrial manufacturers to clean them up.

The Trump administration has threatened to veto the measures, however, calling them "problematic and unreasonable" and a "litigation risk."

But Mededovic said even scientific innovations like hers weren't enough without regulations.

"Elevated levels of PFAS have been found in many public and private water sources, and we need to regulate these compounds," she said. "Companies need to be accountable for what they're producing and releasing."

\section{Link mysterious snippets of DNA to cell growth and tumor suppression}

It's been 80 million years since our evolutionary branch diverged from mice-so why do we share some fragments of DNA that are essentially unchanged?

Why do some strings of genetic code remain virtually unchanged despite tens of millions of years of evolutionary divergence? A newly published study that takes advantage of the gene-editing technique known as CRISPR has found that at least some of those DNA strings are essential to keep healthy cells growing and block the growth of tumor cells.

The research, published today in Nature Genetics, is the "first study finding large-scale importance of these highly conserved elements," senior author Rob Bradley of Seattle's Fred Hutchinson Cancer Research Center said in a news release.

Bradley and his colleagues say unraveling the mysteries of those ultra-conserved elements could lead to new avenues for cancer treatment.

The study started out with a molecular analysis focusing on specialized snippets of DNA known as poison exons. Such snippets regulate the activity of cells by shutting off the production of particular proteins. They've been called "kill switches" for cellular functions. When they go haywire, they can cause maladies such as epilepsy. But when they work the way they're supposed to, they can "poison" the production of undesirable proteins and keep cells on the right track.

Scientists have previously noticed an overlap between poison exons and ultra-conserved elements, but they weren't able to nail down exactly why the ultra-conserved poison exons were so essential. When they knocked out the target exons, one by one, they didn't see much of an effect on cell survival.

The Fred Hutch team took a wider-angle approach: They made use of a CRISPR gene-editing enzyme that was modified with guide-RNA molecules to hunt down and snip out hundreds of poison exons from the genomes of mice, rats and humans $[22,23]$.

Bradley and the study's lead author, Fred Hutch researcher James Thomas, call their technique "paired guide RNAs for alternative exon removal," or pgFARM (pronounced like "pig farm"). "What's unique about what James did is not the approach per se, but that he's doing it in high throughput," Bradley said.

The researchers identified 465 poison exons that were highly conserved between species-as well as another 91 poison exons that weren't so highly conserved. They found that many of the ultra-conserved exons performed similar functions, which explains why knocking out just one of them at a time didn't have much of an effect. But when bunches of the highly conserved exons were snipped out at the same time, the cells died off in a lab dish. 
As a follow-up, the team tested the role of poison exons in live mice that had lung tumors. Many of the exons were essential for normal cell growth, just as they were for the cells in the lab dish. Even more intriguingly, some of the exons had tumor-suppressing effects that were "clinically relevant," the researchers reported.

Tumor-fighting poison exons tended to turn up within the genes that govern RNA splicing, a vital part of the cell's proteinmaking machinery. Those exons may well rein in RNA-splicing factors that could otherwise promote tumor growth $[20,24]$.

Scientists are already working on techniques to fix RNAsplicing problems that contribute to a wide variety of diseases and Thomas is hopeful that ultra-conserved poison exons could point the way to new molecular targets specific to cancer treatment.

"Once you find a target, it's easy to build upon the previous technology that exists for therapeutics," he said.

\section{References}

1. Wu X, Shi J, Fang WX, Guo XY, Zhang LY, et al. Allium vegetables are associated with reduced risk of colorectal cancer: A hospital-based matched case-control study in China. Asia Pac J Clin Oncol. 2019; 15: e132-e141.

PubMed: https://www.ncbi.nlm.nih.gov/pubmed/30790463

2. Anandagoda N, Willis JC, Hertweck A, Roberts LB, Jackson I, et al MicroRNA-142-mediated repression of phosphodiesterase $3 B$ critically regulates peripheral immune tolerance. J Clin Invest. 2019; 129: 12571271.

PubMed: https://www.ncbi.nlm.nih.gov/pubmed/30741720

3. Aria Bendix. Plasma reactor. 2020

4. Thomas JD, Polaski JT, Feng Q, De Neef EJ, Hoppe ER, et al. RNA Isoform Screens Uncover the Essentiality and Tumor-Suppressor Activity of Ultraconserved Poison Exons. Nat Genet. 2020; 52: 84-94. PubMed: https://www.ncbi.nlm.nih.gov/pubmed/31911676

5. Gene found in embryos can cloak cancer from common immunotherapy methods.

6. Fred Hutch scientist on how gold nanoparticles could bring CRISPR to the developing world.

7. Thin metal films loaded with immune cells show promise as cancer therapy.
8. Fred Hutch study discovers how cancer evades immunotherapies, sometimes returning years later.

9. https://www.yahoo.com/news/scientists-mysteriously-unchangedsnippets-dna 043250996.html

10. Ishay-Ronen $D$, Diepenbruck $M$, Kalathur RKR, Sugiyama $N$, Tiede $S$, et al. Gain Fat--Lose Metastasis: Converting Invasive Breast Cancer Cells into Adipocytes Inhibits Cancer Metastasis. Cancer Cell. 2019; 35: 17-32. PubMed: https://www.ncbi.nlm.nih.gov/pubmed/30645973

11. Laura Elizabeth. Converting Cancer Cells to Fat Cells to Stop Cancer's Spread.

12. Elizabeth Chapin, University of Kentucky.

13. Materials provided by University of Manchester.

14. Bafligil C, Thompson DJ, Lophatananon A, Smith MJ, Ryan NA, et al. Association between genetic polymorphisms and endometrial cancer risk: a systematic review. J Med Genet. 2020.

PubMed: https://www.ncbi.nlm.nih.gov/pubmed/32066633

15. Skopec R. New Psychological Weapons Make Targets Hallucinate. J Neuropsychiatry Neurodisorders. 2019.

16. Skopec R. Negative Health Effects of the International Space Station. Int J Res Pharmacy Biosciences. 2019.

17. Skopec R. Fifth, Dark "Force Completely Change Our Understanding of the Universe. Res J Nanoscience Engineering. 2019.

18. Skopec R. Migration: Epidemic Caused By Global Warming and Inequality. Int $\mathrm{J}$ Advancesd Research in Management and Social Sciences. 2018.

19. Skopec R. The Frey Effect Of Microwave Sonic Weapons. Advance Research Journal Of Multidisciplinary Discoveries. 2018.

20. Skopec R. Hybrid Quantum Computing Apocalypse. International Journal of Engineering, Science and Mathematics. 2018.

21. Skopec R. Machine Learning Algorithms Are Pre-Programmed To Humans. Int J Enginee, Sci Mathematics. 2018.

22. Skopec R. Quantum Entanglement Entropy Produces Energy by InfoEntropy Fields Forces. Modern Approaches on Material Science. 2019.

23. Skopec R. Quantum Entanglement Entropy as Karma Lead to New Scientific Revolution. J Enginee Applied Sci Technol. 2019.

24. Skopec R. The Transfiguration With Self-Phase Modulation Effect Of Entanglement In A Plasmatic Moving Frame. IJRDO. J Applied Sci. 2019. 